\title{
L'EXPÉRIENCE COMMUNIELLE DANS LA PRATIQUE NARRATIVE DU JT VIETNAMIEN
}

\author{
Thi Thanh Phuong NguYen-Pochan ${ }^{1}$
}

\begin{abstract}
Cet article s'interroge sur l'articulation de l'expérience communielle avec la dimension informationnelle dans un type du récit spécifique qualifié d'identificatoire. S'inspirant de la narratologie naturelle (Fludernik, 1996), nous envisageons la figure incarnée comme un dispositif de médiation de l'expérience qui renvoie d'une part à un ethos discursif du sujet-expériençant et d'autre part, au régime de croyance qui sous-tend l'adhésion aux valeurs traditionnelles de la culture. Prenant l'exemple de trois récits, retransmis au JT de 19 heures de la télévision vietnamienne, nous examinerons cette articulation à travers la construction de l'ethos discursif de la figure incarnée, le processus d'identification et d'incorporation du téléspectateur à cet ethos, et enfin, le degré d'expérientialité, ou effet de communion, permettant l'adhésion à la communauté imaginaire émanée de l'ethos discursif.
\end{abstract}

« Innombrables sont les récits du monde » (Barthes, 1966, p. 1) : nous dirions, avec Barthes, " innombrables sont les expériences ». L'expérience est constitutive du récit et fait partie intégrante de la pratique narrative. Dans cet article, nous nous intéressons à un type de l'expérience narrative qui permettrait au sujet-réceptant de se projeter dans un monde commun imaginaire et d'expérimenter le sentiment vécu de la communion avec les autres membres de la communauté

1 Thi Thanh Phuong NGuYen-Pochan est docteure en sciences de l'information et de la communication et membre associée au CEMTI (Université Paris 8).

Recherches en communication, $\mathrm{n}^{\circ} 51$ - Article publié le 10/09/2020 
d'appartenance. Notre analyse s'inspire du modèle sémio-pragmatique d'Odin (2011) sur les effets de sens qui sont susceptibles de se produire dans l'espace de communication. Ce modèle est doté d'une valeur heuristique, c'est-à-dire celle d'une " hypothèse dont on ne cherche pas à savoir si elle est vraie ou fausse, mais qu'on adopte seulement à titre provisoire comme idée directrice dans la recherche des faits » (Odin, 2011, p. 19). Les effets décrits dans cet article sont donc des effets potentiels, tels qu'ils se dessinent à partir d'une analyse sémiopragmatique des textes médiatiques.

Nous examinerons l'articulation de l'expérience communielle avec la dimension informationnelle du journalisme narratif, c'est-à-dire la dimension factuelle ou événementielle du récit en lien avec l'actualité, en analysant trois récits retransmis au JT de 19 heures de la télévision vietnamienne (VTV) : le premier relate un rituel bouddhiste; le second est consacré à transmettre la valeur familiale et le rôle des femmes dans la préservation de la tradition; et le troisième raconte les sentiments du peuple vietnamien face au décès du général Võ Nguyên Giáp - une des figures héroïques majeures du Vietnam contemporain.

Ces récits révèlent de nombreuses caractéristiques du journalisme narratif tel qu'il est défini par Vanoost (2013, pp. 152-153) : l'usage « des techniques d'écriture littéraires pour rendre compte d'une histoire réelle »; des personnages qui « déploient leurs actions dans le temps et dans un cadre spatial »; un " narrateur qui possède une voix propre, personnelle »; ou encore la mise en forme de l'histoire " de manière à créer un récit organisé et capable de simuler une forme d'expérience pour ses lecteurs ». En outre, ces récits partagent une même narrativité qui semble pouvoir susciter une adhésion des individus aux valeurs communes de leur propre communauté culturelle, et ce grâce au discours et à la mise en scène (gestuelle, prise de position, scénographie, tonalité, corporalité...) de l'ethos de la figure incarnée, susceptible d'activer le processus d'incorporation ${ }^{2}$ chez le téléspectateur. Les récits du JT de 19 heures que nous analyserons recourent manifestement à deux grands mythes de la tradition vietnamienne : le culte des ancêtres dans ses manifestations sensibles et éthiques (la piété filiale et la tradition familiale) et le culte des héros qui s'entremêle souvent avec le premier car les héros vietnamiens sont aussi considérés comme les ancêtres de la nation.

2 L'usage de ce terme désigne l'effet correspondant à l'inscription d'un corps par l'ethos dans la scénographie. 


\section{L'ethos de la figure incarnée de l'expérience}

Dans la théorie de la «narratologie naturelle » (Fludernik, 1996), l'expérientialité constitue la première matière de la narrativité. Définie comme une « évocation quasi mimétique de l'expérience de la vie réelle $»^{3}$ (Fludernik, 1996, p. 12), l'expérientialité a principalement recours à la figure du sujet-expériençant (experiencer). Selon Fludernik, " il n'y a aucune narrativité sans un sujet-expériençant humain (anthropomorphique) quelconque à un certain degré narratif » (1996, p. 13). Ce sujet désigne une figure incarnée de l'expérience qui est située dans un espace-temps spécifique et dont la perception est cadrée par les paramètres cognitifs " naturels ${ }^{4}$ » propres à l'être humain. Fludernik distingue trois modes narratifs en fonction de trois figures du sujet-expériençant : le mode du narrateur (teller-mode) ; le mode du protagoniste (reflector-mode); et le mode du lecteur ou de la « figure de substitution » (1996, p. 37).

Bien que ce modèle demeure discutable ${ }^{5}$, son caractère heuristique permet d'explorer l'articulation de la dimension informationnelle du journalisme narratif et de l'expérience communielle. D'abord, il déplace le processus de narrativisation du pôle texte au pôle sujetexpériençant et se rapproche ainsi du modèle sémio-pragmatique de la communication : celui-ci explique le processus de la production de sens et d'affects à partir de la co-production du sens par le sujet-émettant et le sujet-réceptant autour d'un même axe de pertinence, désignant les cadres d'interprétation communs et les contraintes pragmatiques propres à une situation de communication concrète (Odin, 2011, pp. 20-21). Ensuite, ce modèle peut servir à analyser la narrativité journalistique dans la mesure où celle-ci est dotée de certains caractères de la narrativité conversationnelle tels que ceux relevant des affects et du pathos, comme en témoignent les processus de spectacularisation et de dramatisation du récit journalistique (Jost \& Spies, 2014). Enfin, ce modèle reste attaché au texte et au contexte historique, comme le sou-

3 Nous traduisons les citations anglaises en langue française.

4 Intention, motif d'action, perceptions de temporalité, d'émotion, de causalité...

5 Caracciolo (2013) s'interroge, par exemple, sur l'interchangeabilité quasi floue entre les deux termes de narrativité et d'expérientialité, ainsi que sur l'ambiguïté définitionnelle du concept d'expérientialité dans le modèle de Fludernik : est-il intrinsèque au récit ou est-il un processus psychologique qui se produit au sein de l'interaction entre le lecteur et le texte? 
ligne l'auteure : « la narrativisation constitue ainsi une frontière processuelle entre le lecteur et le texte, et entre le texte et son historicisation » (Fludernik, 1996, p. 35).

Ce dernier point nous invite à penser l'expérientialité dans sa médiation narrative parce que la figure incarnée ne s'exprime pas de manière spontanée et directe, mais toujours par la médiation d'un ethos. La figure incarnée renvoie à l'ethos discursif d'un « garant» du monde représenté (Maingueneau, 1999, p. 100) : il ne s'agit pas d'une personne empirique, mais d'une « instance subjective incarnée » ou d'une « origine énonciative » que la lecture fait émerger comme une figure dotée d' « un caractère » et d' " une corporalité » (1999, p. 79). L'ethos de la figure incarnée se rapporte plutôt au co-énonciateur (et non destinataire) qu'à l'énonciateur dans une perspective pragmatique : il permet au co-énonciateur de participer physiquement, ou de s'incorporer, dans l'univers de sens du discours, à savoir la " communauté imaginaire de ceux qui adhèrent à un même discours » $(1999$, p. 80$)$.

L'ethos de la figure incarnée renvoie également à un régime de vérité qui structure une vision du monde par le biais d'une expérience du monde commun. Il s'inscrit ainsi dans un "régime de croyance » (Weissberg, 1999, p. 170) susceptible de faire adhérer les membres d'une communauté à un ensemble de valeurs culturelles communes. Dans l'espace médiatique, " croire c'est aussi faire partie d'une communauté, avant d'être un exercice intellectuel. L'adhésion procède d'un mouvement volontaire, ou plutôt nécessaire » (Weissberg, 1999, p. 173). S'il convient de dire qu'aucune expérience ne saurait se produire en dehors des croyances profondes en une vision du monde, l'ethos de la figure incarnée dépasse largement les paramètres purement psychologiques. En ce sens, il vise à renforcer le sentiment communautaire et le lien social et à réaffirmer les valeurs culturelles communes dans l'espace de la communication sociale ou médiatique.

6 Parce que le champ d'expérience est ancré non seulement dans les schémas de vie déjà connus et reconnus (sur le plan psychosocial), mais aussi et surtout dans une vision du monde. Par exemple : ceux qui ne croient pas à la magie des esprits ne pourront pas avoir l'expérience de ceux qui y croient lors de la guérison de maladies, ceux qui ne s'attachent pas à la tradition ne pourront pas accéder à l'expérience de ceux qui vivent ces valeurs... 


\section{L'expérience communielle dans le récit identificatoire}

La notion de récit identificatoire que nous proposons pour qualifier les récits que nous allons analyser s'inspire de la distinction, opérée par Varga (1990), entre la fonction « quotidienne » et la fonction « mythique » du récit postmoderne. Selon l'auteur, les grands récits modernes se transforment en deux formes complémentaires : soit des micro-récits de la vie quotidienne, relevant d'une multitude d'expériences vécues ; soit des récits mythiques qui s'efforcent de remplir le vide que l'homme éprouve suite au déclin des grands récits. C'est la fonction "mythique " du récit qui permet de transmettre les valeurs traditionnelles et communautaires dans les récits que nous allons analyser. La notion de récit identificatoire puise ainsi son efficacité symbolique dans la « force identificatoire du mythe », décrite par Lyotard, qui «n'est pas discutable. On ne peut pas l'expliquer par la mise en représentation spéculaire » (1983, p. 219). Celle-ci rend toujours efficaces les expériences du passé qui demeurent, selon Koselleck, une « force motrice » de l'histoire (1990, p. 317) .

Nous qualifions d'identificatoire le récit qui permet aux récepteurs - ici, aux téléspectateurs - de s'identifier à un monde commun imaginaire. Le récit identificatoire n'est qu'une des différentes catégories du récit médiatique ${ }^{8}$, destinée à « corroborer nos croyances, à nous rassurer sur le plan ontologique » (Varga, 1990, p. 14). Ce récit s'intègre dans le flux des informations. Il se distingue des autres récits médiatiques par sa temporalité propre, sa force symbolique et son champ d'action particulier.

D'abord, le récit identificatoire présente une double temporalité (rétrospective et prospective), dotée d'un sens mythique et non historique, qui se rapporte toujours à un même et unique point d'origine imaginé. Il s'agit d'une temporalité forte, primordiale et anhistorique qui fait revivre et rend présent « le temps prestigieux du commencement » et les « actes exemplaires »(Eliade, 1963, p. 32). Cette temporalité

7 Nous soutenons qu'on peut mettre au même niveau la force identificatoire du mythe et la force motrice de l'histoire dans la mesure où l'histoire est une forme de récit qui recourt largement à la croyance en un mythe (peuple, nation, identité...) ou au schéma narratif mythique.

8 À côté des récits de la vie quotidienne, d'événements imprévus, de connaissances scientifiques, de la santé, de l'environnement... 
renvoie au temps de l'expérience authentique d'une communion sans cesse renouvelable?.

Ensuite, la force symbolique du récit identificatoire tient d'une part au caractère sérieux, solennel, voire sacré qui le distingue d'autres récits ordinaires profanes, et d'autre part, à sa continuité créative avec un passé traditionne ${ }^{10}$. Ce récit tire sa puissance d' « un état d'effervescence » (Durkheim, 1960, p. 603), désignant un état de surexcitation, de passion vive ou de désir de fusion (ou de communion) avec d'autres membres de la communauté d'appartenance identitaire.

Enfin, dans le récit identificatoire, le champ d'action se configure par la structure permanente des événements rituels, en opposition avec la structure changeante (imprévisible) des événements d'actualité imprévus qui surviennent contre toute attente. Alors que la structure temporelle du récit médiatique est généralement irréversible dans la mesure où l'apparition de l'événement est singulière, c'est-à-dire qu'il est doté du temps historique irréversible ; celle du récit identificatoire est réversible, ou circulaire, parce qu'il se configure autour d'un axe du temps fort, consistant non pas à faire apparaître des actes et des paroles dans une succession diachronique, mais à les faire revenir perpétuellement au temps unique.

Ce champ d'action s'inscrit entièrement dans le registre du symbolique où non seulement les significations, mais aussi les acteurs et les énonciateurs sont surdéterminés par une logique transcendante qui transfigure significativement les pratiques et les rapports sociaux. Van Leeuwen (2008, p. 47) parle du processus de «surdétermination » (overdetermination) qui « survient lorsque les acteurs sont représentés comme ceux qui participent simultanément à plus d'une pratique sociale ». Dans un récit identificatoire, la figure incarnée est non seulement un acteur/énonciateur empirique et historique dans un contexte social défini, mais aussi un acteur qui incarne des valeurs et l'expérience d'une figure symbolique et transcendante (les ancêtres mythiques de la nation, par exemple).

Le récit identificatoire vise à faire émerger une expérience agnostique communielle. D'origine religieuse, la communion désigne un état

9 Par exemple, dans le cas de la cérémonie en hommage aux rois Hung - ancêtres imaginaires du peuple vietnamien - chaque année, le vécu mythique du temps d'origine apparaît comme un vécu « symboliquement authentique », à chaque fois unique.

10 Construit par l'idéologie hégémonique des États dynastiques qui se traduit en des mythes, des légendes, des coutumes et des mœurs de la tradition culturelle. 
fusionnel de participation, physique ou mentale, à une performance magique, religieuse ou spirituelle (rite, sacrifice, cérémonie, etc.). Il ne s'agit pas d'ailleurs d'une fusion passive, mais d'une adhésion volontaire et réfléchie de l'individu aux mythes et aux valeurs communes, de son choix délibéré pour un mode d'être communautaire qui lui présente un refuge identitaire rassurant. L'expérience communielle du récit identificatoire donne à voir un vécu sensible et réflexif qui se renouvelle et se rafraîchit à chaque apparition événementielle de la figure incarnée de l'expérience ${ }^{11}$.

L'hypothèse d'une expérience communielle au travers du récit identificatoire part du postulat selon lequel les récits d'actualité dotés d'une fonction « mythique » (Varga, 1990), au-delà de leur dimension informationnelle, cherchent également à répondre au désir rituel. Un des enjeux du récit médiatique consiste en effet, selon Coman (2005), à faire revivre et ressentir une histoire mythique du monde commun, ce qui explique en grande partie la prégnance du modèle narratif archétypal (Lule, 2005 ; Lits, 1997).

\section{L'analyse des trois récits du JT de 19 heures}

Les trois récits que nous analyserons ont été sélectionnés, parmi un corpus plus large de séquences du JT vietnamien de 19 heures, suivant trois critères : premièrement, ils présentent les caractéristiques du type de récit que nous qualifions d'identificatoire (temporalité, champ d'action, force symbolique, expérience communielle) et ils recourent tous aux valeurs mythiques de la culture vietnamienne; deuxièmement, ils présentent des récits complets suivant le modèle du journalisme narratif ; et troisièmement, ces trois récits fournissent des exemples concrets pour les trois modes narratifs basés respectivement sur la figure du narrateur (ici le journaliste), la figure du protagoniste et la figure de substitution (ici le téléspectateur) tels qu'ils sont présentés dans le modèle de Fludernik.

La dimension expérientielle de ces récits journalistiques sera examinée à travers :

11 Cette articulation de la structure intelligible et de l'expérience sensible dans le récit identificatoire renvoie à la théorie straussienne sur le bricolage de la pensée mythique en combinant structure permanente (nécessaire) avec événement (contingent) (Lévi-Strauss, 1962, pp. 32-33). 
- la construction de l'ethos discursif de la figure incarnée (experiencer) ;

- la façon dont cet ethos est susceptible de susciter un processus d'incorporation du téléspectateur ;

- les trois modes narratifs d'expérientialité qui en découlent et leur capacité à pousser le téléspectateur à adhérer à la communauté imaginaire émanée de l'ethos de la figure incarnée - cette adhésion reposant sur le régime de croyance qui sous-tend les valeurs traditionnelles et identitaires de la culture.

\subsection{Le récit au mode du narrateur}

Le récit intitulé « La cérémonie "la rose épinglée à la poitrine" »", retransmis le 31 août 2012, est introduit dans le JT comme un événement inscrit dans l'actualité :

Aujourd'hui, c'est le jour de la pleine lune du $7^{\mathrm{e}}$ mois lunaire [...] C'est l'occasion pour chacun des Vietnamiens de témoigner sa gratitude ${ }^{13}$, de rendre les bienfaits et de faire acte de piété filiale, conformément à l'esprit traditionnel de la nation vietnamienne. Dans le rituel Vu Lan, il y a une cérémonie spéciale appelée La rose épinglée à la poitrine consacrée à la méditation et à la commémoration des bienfaits de ceux qui nous ont mis au monde et élevés.

Cette description factuelle, employant le langage cultuel, se rapporte à des schémas culturels connus de tous et inscrit ainsi ce récit journalistique dans le régime d'événement rituel. Par la suite, la narration se déploie de manière à rendre sensibles ces valeurs abstraites de la culture. Dès la première scène, la diégèse fait entrer le téléspectateur dans un univers rituel par le biais des images religieuses (les pratiquants bouddhistes portant les tuniques marrons et épinglant les roses à la poitrine, les prières...), d'une musique mélodramatique, d'une voix étranglée récitant un poème sur l'amour parental, des larmes qui coulent, etc. La diégétisation fonctionne non seulement grâce à la succession des images-sons, mais aussi par le biais de marqueurs discursifs

12 Nous traduisons les titres, introductions et textes des récits.

13 Nous mettons en italiques les descriptions cultuelles et traditionnelles. 
relevant du champ cultuel : « Il est difficile pour chacun de refouler ses sentiments au moment où son âme s'apaise et où il présente l'offrande sincère de son âme empreinte de reconnaissance à ceux qui l'ont mis au monde et élevé. » La narration joue ensuite sur le terrain de l'expérience en donnant trois types d'expérience :

Dans la cérémonie La rose épinglée à la poitrine, ceux dont les parents sont encore vivants épinglent une rose rouge afin de ressentir le bonheur qu'ils ont et de se rappeler à leur devoir de faire plaisir aux parents ; une rose blanche aux orphelins pour qu'ils commémorent leurs parents défunts; et une rose mauve à ceux dont un des parents est décédé.

Le rituel $V u$ Lan est censé être le moment de rassemblement des individus partageant les mêmes valeurs et croyances en une communion spirituelle avec les personnes défuntes. La narration souligne ce sentiment d'appartenance comme suit :

$V u$ Lan est la saison de la piété filiale - saison du battement de cour d'amour et saison du rassemblement pour la reconnaissance. L'esprit de chacun s'enrichit par la piété filiale et la concorde fraternelle, et par la reconnaissance envers le noyau essentiel de la morale humaine.

Trois témoins sont ensuite invités à exprimer leur amour parental et la signification de cette cérémonie dans la vie moderne. À la fin du récit, c'est le journaliste-narrateur qui témoigne ce sentiment et cette morale par l'insertion de sa voix-in qui se présente ici comme une expérience intra-diégétique. L'introduction du corps et de la voix du narrateur dans le texte médiatique peut être considérée comme une stratégie de proximité « corporalisante » (Ringoot \& Rochard, 2005, p. 78) visant à montrer un journaliste-acteur en train d'agir dans la trame narrative du récit. Or, dans ce cas particulier, il n'y a aucune image préalable du narrateur qui permet une inscription de son corps dans la scénographie du récit : son entrée dans la scène est faite d'une manière abrupte. Quant à sa voix, le narrateur adopte, dans la première partie de son énoncé, le statut de je-origine réel (Jost, 1995, p. 166) qui joue sur le registre personnel en énonçant à la première personne : 
Dans la cérémonie d'aujourd'hui, je suis ravie de pouvoir choisir la rose rouge, mais mon sentiment à l'égard des parents, tout comme celui des centaines de personnes présentes, est indicible.

Mais il abandonne rapidement ce statut pour adopter la parole de l'énonciateur surdéterminé :

Combien de larmes maternelles ont coulé, combien de sueurs paternelles ont été versées pour élever les enfants? Dans la vie moderne, de tels moments d'apaisement sont extrêmement précieux pour que chacun puisse autoperfectionner sa voie morale de piété filiale.

Malgré le recours au pronom je, l'expérience du narrateur apparaît totalement théorique (au sens de Jost, 1995) : elle relève d'un ethos abstrait qui n'est pas capable d'évoquer une expérience de la vie réelle. C'est une voix de ventriloque, qui prend une voix qui n'est pas la sienne, comme celle d'une marionnette, pour transmettre les valeurs et idées abstraites de la culture sans parvenir à les faire vivre au travers d'une expérience personnelle.

\subsection{Le mode narratif basé sur le protagoniste}

L'introduction du second récit qui s'intitule « Le repas familial », retransmis le 28 juin 2014, combine deux registres de faits : l'un relève de l'actualité (de l'événement contingent) et l'autre relève de données culturelles préexistantes (de la structure permanente) :

Le dîner, emblème de la cohésion familiale, est le moment où les membres discutent, où les grands-parents orientent la conduite de leurs petits-enfants et leur transmettent les belles coutumes et traditions de la famille et de la société. Cependant, selon des statistiques récentes, dans les grandes zones urbaines du Vietnam, les membres de la famille sont régulièrement absents au dîner dans près d'un tiers des foyers [...]. Selon plusieurs spécialistes de la culture, quel que soit le niveau de développement de la société, le dîner en commun demeure le miroir reflétant le bonheur familial. C'est la raison pour laquelle la Journée 
consacrée à la famille vietnamienne cette année a comme thème : Le repas familial chaleureux et plein d'amour.

La narration choisit un angle d'approche différent du premier récit : elle présente au téléspectateur une personne physique et réelle, bien située dans l'espace-temps concret. Le personnage principal est Lan Hương, créatrice de tuniques traditionnelles áo dài, qui joue ici le rôle de « femme gardienne du feu du foyer ». La métaphore de la « gardienne du feu » est tirée d'une représentation sociale largement partagée : la fonction essentielle de la femme dans la société vietnamienne traditionnelle est de s'occuper de la cuisine. La protagoniste incarne à la fois la modernité (de par son activité professionnelle) et la tradition (du fait de son rôle dans le foyer, mais aussi du fait que son métier s'inspire de l'esthétique traditionnelle).

Le récit débute par des séquences d'images illustrant ses activités dans la journée : faire les courses, cuisiner, discuter avec les enfants, etc. Ces images sont accompagnées de description factuelle : « une journée de Lan Hương - la créatrice de tuniques áo dài-commence souvent par des courses tôt le matin afin de préparer le repas familial »; mais aussi de description axiologique : « sa famille se compose de trois générations, chacune a ses propres préoccupations. Ainsi, en tant que gardienne du feu du foyer, elle prépare toujours le repas quotidien afin d'entretenir les relations avec les parents et d'écouter les enfants ». Cette femme est toujours vêtue de l'áo dài, symbole de tradition. Elle livre ensuite, dans une voix-in, son expérience au téléspectateur : « Le repas est un moment exceptionnel et significatif où les membres de la famille échangent, partagent, s'encouragent, voire se critiquent. Je le trouve très important dans notre vie moderne. "

L'expérience de Lan Hương est ici susceptible d'interpeller directement celle de la téléspectatrice - et, plus indirectement, celle du téléspectateur : grâce à un « recadrage événementiel » vraisemblable (Esquenazi, 2002, p. 78), il y a un effet miroir avec la vie réelle des femmes vietnamiennes. Son discours paraît crédible et sincère car il s'énonce à partir d'un ethos (son corps et sa voix) qui se construit tout au long du récit et qui survient d'une scénographie établie et familière au téléspectateur : celle de la vie quotidienne des femmes vietnamiennes suivant la conception traditionnelle.

Le récit ne se temporalise pas : il n'explique pas, par exemple, comment cette femme organise sa journée afin de pouvoir remplir ses devoirs au sein du foyer (traditionnellement assignés aux femmes), et 
aussi ses activités professionnelles et personnelles. Au contraire, le récit retourne au point de départ qui est la valeur précieuse et centrale de la famille. Le téléspectateur entend, à la fin du reportage, une voix-off, celle de la protagoniste qui semble planer sur les images, accompagnée d'une musique paisible : " Si nous nous acharnons à travailler, à nous focaliser sur nos relations mondaines, et oublions que le but final est de nous arrêter à notre embarcadère, tout ce que nous faisons risque un jour de perdre de son sens ». L'énonciatrice prend le statut surdéterminé de celle qui parle au nom de toutes les femmes («nous ») attachées aux valeurs familiales, faisant ainsi revivre l'attitude féminine millénaire. Cependant, puisque sa voix surdéterminée s'appuie sur un ethos déjà confirmé, elle permet de faire entrer d'autres femmes dans cet univers traditionnel, d'autant plus qu'elle utilise la métaphore de « notre embarcadère », qui apparaît comme le symbole d'un refuge rassurant pour les femmes dans une vie moderne devenue plus complexe que la vie traditionnelle. Cette expérience de la protagoniste apparaît donc partageable et expérimentable en tant qu'expérience culturelle commune, car elle se livre à travers une voix culturellement crédible et naturelle, et non une voix de ventriloque d'empilement de valeurs.

\subsection{Le mode narratif basé sur l'expérience du téléspectateur}

Le dernier récit intitulé « L'amour du peuple pour le général Võ Nguyên Giáp » (VNG) se distingue des deux premiers récits par son caractère événementiel qui consiste dans une rupture avec la vie ordinaire causée par l'annonce du décès du général VNG au JT le 5 octobre 2013. Le général VNG est considéré par les Vietnamiens comme une personne possédant toutes les qualités du héros idéal selon la conception confucéenne (patriotisme, vaillance, combativité, noblesse et humanisme, etc.). Incarnant la morale intacte d'un héros exemplaire de soldat du vieux Hô, il est respecté par le peuple et l'armée. Comparé aux dirigeants corrompus d'aujourd'hui, VNG est le miroir d'un passé glorieux qui se reflète dans un présent honteux (Currey, 2005 ; Guillemot, 2013). Au respect de la vertu, il faut ajouter le facteur de l'âge (décédé à l'âge de 103 ans) que la tradition gérontologique vietnamienne consacre. Dans ce sentiment multiple et dans la croyance populaire, sa longévité est un signe favorable pour le destin national. Ainsi, la mise en scène de son décès au JT visait à créer une expérience bou- 
leversante pour le public ${ }^{14}$, en se cristallisant autour de la perte d'une figure incarnée de la morale exemplaire de la nation (Nguyen-Pochan, 2017).

La narration de ce récit se caractérise par la reprise, par le journaliste-narrateur, de discours émotifs et poétiques du public. La première séquence d'images, d'une durée de 9 secondes sans commentaire, montre une page Facebook où est postée une photographie du général avec son petit-fils, accompagnée d'une musique lyrique. Suite à cette entrée en douceur, la voix-off du narrateur s'élève :

19 heures, minuit, 2 heures du matin, la nuit dernière a été une nuit blanche pour la communauté des internautes. Les images du général bien-aimé et respecté sont omniprésentes sur les pages des réseaux sociaux. Et aussi nombreux sont les partages : «Ô grand-père, mon cour étouffe. L'automne vient d'arriver, c'est maintenant que vous partez. Après une vie pleine, digne, morale et emplie de deux mots : amour du peuple. »

La voix-off tremblotante du narrateur récite le poème d'un auteur anonyme circulant sur Internet sur un fond musical mélancolique. Au travers d'emprunts aux poèmes composés par les internautes, ce récit recouvre l'expérience des jeunes vietnamiens dont la présence sur Internet est la plus audible. Il fait émerger la voix d'un tiers symbolique, collectif, poétique et sentimental : «Eux, ces jeunes gens qui sont nés après la guerre, ne connaissent le général VNG qu'au travers de la presse, des écrits historiques et des conversations de leurs grandsparents. \ Ce qui est intéressant dans ce récit, c'est que la virtualité de l'ethos du tiers symbolique se base sur une expérience réelle de la vie virtuelle sur les réseaux sociaux qui est partageable par n'importe quel utilisateur d'Internet. La scénographie qui lui donne corps est maintenue par les deux témoignages qui sont directement rapportés : «Les journaux en ligne et les pages Facebook en ont beaucoup parlé, j'ai voulu passer ici pour vérifier si c'était vrai »; « Tout le monde connaissait son état de santé, mais son décès est cruel ». Ces témoignages, et les

$14 \mathrm{La}$ presse internationale s'est fait l'écho de cette expérience bouleversante : https://www.rtbf.be/info/monde/detail_vietnam-une-foule-immense-dit-adieu-augeneral-giap-heros-de-guerre?id=8111674 et https://www.bbc.com/news/world-asia-24516186 
commentaires suivants, renforcent le sentiment de sidération provoqué par cet événement :

La nuit dernière, cette nuit et la nuit prochaine, dans cette rue, beaucoup s'arrêteront pour y attendre patiemment une silhouette solide. Ces vers sont partagés par un jeune ami dans la nuit : "Accompagnons l'Homme qui retourne là-bas, au bout du Ciel. Là où se trouvent l'Oncle Hô, les fondateurs de la nation et aussi Trần Hưng Đạo, Lê Lọi et Quang Trung. Dans la Patrie éternelle, les Hommes se succèdent. La nation perdure car elle fait naître les Héros. »

Le monde représenté passe de l'espace virtuel d'Internet vers la rue où vivait le général. Plusieurs personnes, l'air désolé, se rassemblent ou passent devant sa maison dans une attente anxieuse de nouvelles : ils veulent s'assurer de la véracité de son décès. Les jeunes gens, tristes et soucieux, évoquent la mort de VNG comme celle de leur grand-père. La séquence se referme avec la lune et les branches d'arbre de la rue hanoïenne, la maison où repose son âme, puis la récitation, par une voix tremblotante, de vers fraîchement composés en son hommage. La sidération et le sentiment de perte trouvent ainsi refuge dans un monde familial, intime et paisible. Dans ce récit, la volonté de communion, spirituelle et sentimentale, s'exprime pleinement : la communion, qui cimente les Vietnamiens dans une solidarité de douleur, se révèle aussi entre eux et l'esprit du général défunt. Cette communion, à la fois naturelle et sincère au niveau individuel (car elle touche au vécu intime), apparaît sacrée et effervescente au niveau collectif (car elle touche à une valeur intouchable de la communauté d'appartenance).

\section{Conclusion}

Ces trois exemples sont certes insuffisants pour tirer des conclusions générales sur le fonctionnement de chacun des trois modes narratifs. Ils permettent cependant d'observer, dans une perspective sémio-pragmatique, les formes de communion différentes qui peuvent être générées par ces modes narratifs dans le récit identificatoire. D'abord, le mode d'expérientialité dépend de la relation entre l'ethos et le téléspectateur: à quel point cet ethos interpelle-t-il le vécu du téléspectateur? Il dépend ensuite de la possibilité de participation «physique » du téléspectateur, 
c'est-à-dire de son incorporation dans la trame narrative en tant que tiers symbolique. L'effet de communion, tel que nous l'entendons, ne se produit pleinement que lorsque la frontière entre l'expérience individuelle et l'expérience communautaire se trouve effacée, lorsque ce que l'individu ressent personnellement rejoint son sentiment d'appartenance à la communauté culturelle. Tel est le cas du récit sur le décès du général Võ Nguyên Giáp dans lequel la douleur personnelle d'un individu entre en communion avec le chagrin collectif.

Enfin, dans les trois récits analysés, les formes de communion paraissent dépendre de la nature événementielle du récit : la séquence qui est liée à la plus grosse rupture dans l'expérience quotidienne du public semble en effet pouvoir susciter le sentiment de communion le plus intense. Tel est le cas pour les évènements comme des guerres, des attaques terroristes, des catastrophes naturelles, ou encore des moments liminaires de la vie collective (à l'instar des manifestations récentes des Gilets Jaunes en France). Ainsi, l'expérience communielle paraît étroitement liée à la dimension informationnelle. Ce sont souvent ces circonstances extraordinaires de la vie collective qui créent l'opportunité unique de faire revivre un mythe, et cela dans une expérience communielle de la société. C'est d'ailleurs une logique essentielle de fonctionnement du mythe : celui-ci est avant tout un vécu événementiel et non simplement une expérience répétitive du rituel (Eliade, 1963, pp. 32-33).

\section{Références}

Barthes, R. (1966). Introduction à l'analyse structurale du récit. Communications 8 , 1-27.

Caracciolo, M. (2013). Experientiality. Dans P. Hühn et al. (Éd.), The Living Handbook of Narratology. Hambourg : Hamburg University Press. Disponible à : http://www. lhn.uni-hamburg.de/article/experientiality

Coman, M. (2005). News Stories and Myth - The Impossible Reunion? Dans M. Coman \& E. W. Rothenbuhler (Éd.). Media Anthropology (pp. 111-120). Londres : Sage Publications.

Currey, C. B. (2005). Victory at Any Cost. The Genius of Vietnam's Gen. Vo Nguyen Giap, Washington : Brassey's.

Durkheim, E. (1960). Les Formes élémentaires de la vie religieuse. Coll. Quadrige. Paris : PUF (quatrième édition).

Eliade, M. (1963). Aspects du mythe. Paris : Gallimard.

Esquenazi, J.-P. (2002). L'Écriture de l'actualité. Pour une sociologie du discours médiatique. Grenoble : Presses universitaires de Grenoble.

Fludernik, M. (1996). Towards a "Natural" Narratology. Londres : Routledge. 
Guillemot, F. (2013). Vo Nguyen Giap, le « Napoléon vietnamien », héros intangible. Mémoires d'Indochine. Disponible à : https://indomemoires.hypotheses.org/11484 Jost, F. (1995). Le feint du monde. Réseaux 13(72-73), 163-175.

Jost, F. \& Spies, V. (2014). L'information à la télévision, un spectacle ? Revue française des SIC 5. Disponible à : https://journals.openedition.org/rfsic/1123

Koselleck, R. (1990). Le Futur passé. Contribution à la sémantique des temps historiques. Paris : EHESS.

Lévi-Strauss, C. (1962). La Pensée sauvage. Paris : Plon.

Lits, M. (1997). Le récit médiatique : un oxymore programmatique ? Recherches en Communication 7, 37-59.

Lule, J. (2005). New As Myth. Daily News and Eternal Stories. Dans M. Coman \& E.W. Rothenbuhler (Éd.), Media Anthropology (pp. 101-110). Londres : Sage Publications.

Lyotard, J-F. (1983). Le Différend. Paris : Les Éditions de Minuit.

Maingueneau, D. (1999). Éthos, scénographie, incorporation. Dans R. Amossy (Éd.), Images de soi dans le discours. La construction de l'ethos (pp. 75-100). Lausanne : Delachaux et Niestlé.

Nguyen-Pochan, T.T.P. (2017). Quand la télévision devient le centre sacré de la communauté identitaire. La médiatisation des événements cérémoniels à la Télévision vietnamienne. ESSACHESS 2(20), 53-75.

Odin, R. (2011). Les Espaces de communication. Introduction à la sémio-pragmatique. Grenoble : Presses universitaires de Grenoble.

Ringoot, R. \& Rochard, Y. (2005). Proximité éditoriale : normes et usages des genres journalistiques. Mots 77, 73-90.

Van Leeuwen, T. (2008). Discourse and Practice. New Tools for Critical Discourse Analysis. Oxford : Oxford University Press.

Vanoost, M. (2013). Journalisme narratif : proposition de définition, entre narratologie et éthique. Les Cahiers du journalisme 25, 140-160.

Varga, A. K. (1990). Le récit postmoderne. Littérature 77, 3-22.

Weissberg, J.-L. (1999). Dispositif de croyance. Hermès 25, 169-178.

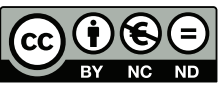

«Attibution - pas d'utilisation Commerciale - Pas de Modification 4.0. International» (CC BY-NC-ND) 